\section{Time for a preventative strategy for TB in the UK: further evidence for new entrant screening in primary care}

\author{
Onn Min Kon
}

In 2012, a total of 8751 cases of TB in the UK were reported at a rate of 13.9 cases per 100000 -this figure is on the background of an increase over two decades in notifications and rates with no evidence of any reduction since peaking in $2005 .^{1}$ This rise has been largely attributed to TB in the foreign-born, with $73 \%$ of all cases of TB being born in high-incidence countries. By contrast, the cases of TB in the UK born have been static over the last decade. Given that these cases are largely due to reactivation disease, ${ }^{2}$ a strategy of $\mathrm{TB}$ control purely based on detection and treatment of active pulmonary disease is unlikely to cause a significant change in these statistics. Hence, despite the change in TB screening to the port of exit, this is also unlikely to be effective given that the major burden of latent disease will not be detected by plain radiology.

A strategy of screening in the USA has been credited with the decreasing rates of TB there which is in stark contrast to the UK statistics. They have a rigorous approach with pre-entry screening followed by evaluation again after arrival in those with treated active but also latent TB. ${ }^{3}$ Our completion rates are improving and nearly in line with WHO standards ${ }^{1}$ and, hence, there needs to be a significant change in our approach as further improving treatment completion in itself is unlikely to cause a significant reversal in rates. Given the complexities of this disease and the multiple components of prevention, detection, treatment and engagement; tackling TB in the UK will continue to need a multipronged approach. In addition to addressing the high rates in the most vulnerable and the 'hard to reach', the current missing component is the systematic implementation of new entrant screening. Previous audits of practice in Europe and the UK have paradoxically shown that the services with the highest rates of disease are least able to

Chest and Allergy Clinic, St Mary's Hospital, Imperial College Healthcare NHS Trust, London, UK

Correspondence to Dr Onn Min Kon, Chest and Allergy Clinic, St Mary's Hospital, Imperial College Healthcare NHS Trust, Praed Street, London W2 1NY, UK; onn.kon@imperial.nhs.uk offer this. ${ }^{4}$ Notably, new entrant screening has been part of National Institute of Health and Care Excellence (NICE) recommendations since 2006 but with little evidence in reality of any systematic strategy or dedicated funding to accompany this being

Panchal et $a l^{6}$ have performed a retrospective analysis over 11 years of all recorded registrations of foreign born individuals in primary care and also foreign born TB notifications in Leicestershire and demonstrated that $60 \%$ of their active cases of foreign born cases could have been identified and potentially prevented with chemoprophylaxis. Notably, they also demonstrate that the uptake of GP registration differs dependent of the country of origin with the Indian Subcontinent cases being the most likely to register and notably less so in individuals from Sub-Saharan Africa. There also appeared to be an association of TB cases with later registration.

Despite the limitations of not being able to verify the applicability of this approach in other conurbations and areas in the UK, their data again confirms a threshold of 150 per 100000 incidence in the country of origin is likely to be most effective. This consolidates the findings of other recent publications on the optimal cost effectiveness of a threshold at 150 per $100000 .^{78}$ As with other aspects of TB care a regional 'whole system' view is important and needs to continue to be a focus of public health overview to inform collaborative commissioning given the potential for there to be fragmentation of TB care in the new commissioning environment. NICE is currently re-evaluating its guidance but has varied in its approach initially in 2006 advocating screening at rates of 500 per 100000 and Sub Saharan Africa only and then decreasing this threshold to 40 per 100000 in $2011 .^{5}$ Hence a revision of the threshold of a 40 per 100000 incidence and modelling of which groups to screen $^{9}$ should be re-examined critically as our use of available resource is likely to be limited.

Importantly this paper indicates that primary care would be an appropriate venue to evaluate these cases at new implemented in the community. ${ }^{5}$ registration and has previously been shown to be a viable option for such screening. ${ }^{8} 10$

As the paper by Panchal et $a l^{6}$ illustrate, ${ }^{11}$ there needs to be community engagement to improve registration rates and reduce the time to registrations in all ethnic groups-a 'whole system' solution is most likely to deliver a real decrease in $\mathrm{TB}$ rates and community engagement is critical. Hence the involvement of third sector organisations in addition to the appropriate funding for new entrant screening are critical in delivering a strategy to reduce rates of TB in the UK. It will also be important to consider screening for other blood borne viruses as well as TB in terms of wider migrant health given the potential to screen individuals by blood tests rather than by the traditional tuberculin skin test following the advent of interferon gamma release assays.

Although several outstanding issues still need exploring such as the actual uptake of screening and chemoprophylaxis, unless there is a systematic change in our approach to latent TB treatment, it is likely we will not see a significant reduction in the rates of TB in the UK and risk remaining an 'outlier' in Western Europe. ${ }^{12}$

Competing interests None.

Provenance and peer review Not commissioned; internally peer reviewed.

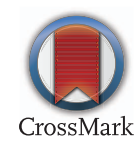

To cite Kon OM. Thorax 2014;69:305-306.

Published Online First 2 January 2014

Thorax 2014:69:305-306.

doi:10.1136/thoraxjn-2013-204777

\section{REFERENCES}

1 Public Health England. Tuberuclosis in the UK 2013 report. London: Public Health England. 2013. http:// www.hpa.org.uk/webc/HPAwebFile/HPAweb_Cl 1317139689583

2 Love J, Sonnenberg P, Glynn JR, et al. Molecular epidemiology of tuberculosis in England, 1998. Int J Tuberc Lung Dis 2009;13:201-7.

3 Liu Y, Weinberg MS, Ortega LS, et al. Overseas screening for tuberculosis in U.S.-bound immigrants and refugees. N Engl J Med 2009;360:2406-15.

4 Pareek M, Abubakar I, White PJ, et al. Tuberculosis screening of migrants to low-burden nations: insights from evaluation of UK practice. Eur Respir J 2011;37:1175-82.

5 National Collaborating Centre for Chronic Conditions (UK), Centre for Clinical Practice at NICE (UK). Tuberculosis: clinical diagnosis and management of tuberculosis, and measures for its prevention and control. London: National Institute for Health and Clinical Excellence (UK), 2011.

6 Panchal RK, Browne I, Monk P, et al. The effectiveness of primary care based risk 


\section{Editorial}

stratification for targeted latent tuberculosis infection screening in recent immigrants to the UK: a retrospective cohort study. Thorax 2014;69: 354-62.

7 Pareek M, Watson JP, Ormerod LP, et al. Screening of immigrants in the UK for imported latent tuberculosis: a multicentre cohort study and cost-effectiveness analysis. Lancet Infect Dis 2011;11:435-44.

8 Pareek M, Bond M, Shorey J, et al. Community-based evaluation of immigrant tuberculosis screening using interferon $\gamma$ release assays and tuberculin skin testing: observational study and economic analysis. Thorax 2013;68:230-9.

9 Kruijshaar ME, Abubakar I, Stagg HR, et al. Migration and tuberculosis in the UK: targeting screening for latent infection to those at greatest risk of disease. Thorax 2013;68:1172-4.

10 Griffiths C, Sturdy P, Brewin P, et al. Educational outreach to promote screening for tuberculosis in primary care: a cluster randomised controlled trial. Lancet 2007;369:1528-34.
11 Stagg HR, Jones J, Bickler G, et al. Poor uptake of primary healthcare registration among recent entrants to the UK: a retrospective cohort study. BMJ Open 2012;2:e01453.

12 European Centre for Disease Prevention and Control (ECDC)/WHO Regional Office for Europe. Tuberculosis surveillance and monitoring in Europe 2013.

Stockholm: ECDC, 2013. http://www.ecdc.europa.eu/ en/publications/Publications/Tuberculosis-surveillancemonitoring-2013.pdf 